\title{
CASE REPORT: ASSOCIATION BETWEEN LYMPH NODE TUBERCULOSIS AND BREAST CANCER
}

Marina Fontes Medeiros¹, Gustavo Lanza de Melo¹, Thássia Mariz de Melo¹, Rachel Saraiva Teatini Selim de Sales¹, Janaina Cotta Rodrigues Ferreira'

IInstituto Mário Penna - Belo Horizonte (MG), Brazil.

Introduction: Lymph node tuberculosis is the most common extra-lung presentation of tuberculosis, responsible for $43 \%$ of peripheral lymphadenopathies in developing countries. The coexistence between lymph node tuberculosis and breast cancer is rare, ranging from $0.1 \%$ to $4.9 \%$.Objective: To present a case of axillary lymph node tuberculosis due to its rare association with breast cancer. Methods: We have investigated the case of a 48-year-old woman from Congonhas, Minas Gerais, Southeastern Brazil, who presented a palpable nodule in the junction of the right upper quadrants with two years of progression, category 4B of the Breast Imaging Reporting and Data System (BI-RADS) on mammography and ultrasound, with core needle biopsy compatible with benignancy. No axillary lymphadenopathy was identified. Case report: The patient underwent resection of the right breast nodule with safety margins due to disagreement between biopsy and imaging tests. Anatomopathological examination was consistent with luminal B invasive ductal carcinoma, measuring 1.6 $\mathrm{cm}$. The patient was submitted to sentinel lymph node biopsy using patent blue in the right axilla. Anatomopathological analysis revealed tuberculous lymphadenitis. Chest computed tomography showed pulmonary nodules. The patient received adjuvant radiotherapy and tamoxifen, as well as antituberculous antibiotics, with regression of pulmonary nodules. The final staging was pT1cN0M0- IA. Discussion: Most cases of coexistence between these diseases involve tuberculous lymphadenitis with or without neoplastic lymph node involvement. Some reports indicate that the involvement by tuberculosis does not prevent neoplastic proliferation. Before starting chemotherapy, tuberculosis must be treated to avoid the immunosuppressive effect that can cause a spread of tuberculosis. Conclusion: Despite the rare coexistence of these diseases, we should not rule out this possibility, especially in endemic tuberculosis areas. Also, an accurate diagnosis prevents incorrect staging and can spare the patient from a more aggressive treatment. 